\title{
Unkomplizierte Harnwegsinfekte
} Im Wettlauf mit den Resistenzen
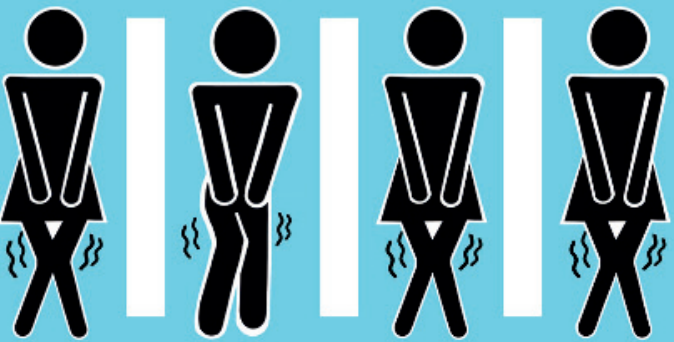

Die empirische Therapie von Harnwegsinfekten bereitet zunehmend Probleme, weil gängige Antibiotika unwirksam werden. Bis neue Behandlungskonzepte entwickelt sind, muss sich die Therapie an der aktuellen Resistenzlage orientieren.

Uropathogene Mikroorganismen können entsprechend ihren Virulenzfaktoren eine Zystitis, eine Pyelonephritis, eine Bakteriämie oder eine Urosepsis verursachen. Dabei wird das Eindringen der Bakterien in den Harntrakt durch verschiedene Faktoren begünstigt. Jede zweite Frau, schreibt Prof. Walter H. Hörl von der Medizinischen Universitätsklinik III, Wien, in der Zeitschrift „Internist“, entwickelt mindestens einmal im Leben einen Harnwegsinfekt, ein Viertel davon immer wieder. Vor allem in der Menopause, wenn sich die Vagi-

\begin{tabular}{l|l}
\hline Tab. 1 & $\begin{array}{c}\text { Diagnose } \\
\text { Harnwegsinfekt }\end{array}$ \\
\hline Faktor & \multicolumn{1}{c}{ Punkte } \\
\hline Nitrit positiv & 2 \\
Leukozyturie' & 1,5 \\
\hline Hämaturie & 1 \\
\hline Dysurie & 1 \\
\hline Nykturie & 0,5 \\
\hline $\begin{array}{l}\text { Auswertung: } \\
\text { Je höher die Punktzahl, desto } \\
\text { wahrscheinlicher ist ein Harnwegsinfekt. }\end{array}$ \\
\hline $\begin{array}{l}\text { 'Leukozyturie im Zusammenhang mit der } \\
\text { Urinmenge interpretieren. } \\
\text { (W. H. Hörl, Internist 2011, 52:1026) }\end{array}$
\end{tabular}

nalflora aufgrund des lokalen Östrogenmangels verändert oder nach einer Antibiotikatherapie haben harnpathogene Keime leichtes Spiel.

\section{Jede dritte Diagnose falsch}

Da Schätzungen zufolge nahezu jede dritte Diagnose falsch ist, soll ein Punktesystem die Sicherheit verbessern (- Tab. 1). Ergänzend können Keimzahlbestimmungen und Antibiogramme sowie ggf. Hemmstofftests durchgeführt werden.

Bei bis zu 42\% der Patientinnen mit einer unkomplizierten Zystitis wurde eine spontane Heilung beobachtet. Der Autor empfiehlt dennoch eine Antibiotikatherapie etwa mit Pivmecillinam 2x $400 \mathrm{mg}$ für drei Tage oder 2x $200 \mathrm{mg}$ für sieben Tage, aufgrund niedriger Resistenzraten und guter Verträglichkeit alternativ die Einmalgabe von Fosfomycin-Trometamol. Auch Nitrofurantoin kann, trotz beträchtlicher Nebenwirkungen, gegeben werden, so Hörl. Die deutsche S3-Leitlinie sieht zudem vor, dass Fluorchinolone nur noch bei lokalen Resistenzraten unter 20\% verwendet werden und nur dann, wenn Präparate der ersten Wahl nicht eingesetzt werden können. In diese Kategorie fallen für ansonsten gesunde, nicht schwangere Frauen auch Cotrimoxazol, Trimethoprim, Cephalosporine und die Aminopenicilline in Kombination mit einem Betalaktamaseinhibitor.

Eine Pilotstudie aus dem Jahr 2010 hat gezeigt, dass 58,3\% der Frauen, die nur mit 3x 400 mg Ibuprofen/Tag behandelt worden waren, am vierten Behandlungstag beschwerdefrei waren. Erhielten die
Patientinnen 2x 250 mg Ciprofloxacin und 1x Placebo/Tag, waren im gleichen Zeitraum 51,5\% ohne Beschwerden. Allerdings wurden unter Ibuprofen tendenziell mehr Zweitbehandlungen erforderlich. Frauen ohne Kontraindikation, so der Autor, könnten möglicherweise von einer Kombination aus Schmerzmittel und Antibiotikum profitieren, da damit sowohl Symptome als auch Keime eliminiert werden.

\section{Neue Therapien in Sicht?}

Angesichts zunehmender Antibiotikaresistenzen setzt man vor allem auf neue Therapiekonzepte. Interessant erscheinen derzeit z. B. Metaboliten des körpereigenen Stoffwechsels. In ihrer Gegenwart werden höhere Konzentrationen an Aminoglykosiden in die Zelle aufgenommen. Besonders gegen intrazelluläre Mikroorganismen, die sich einer konventionellen Antibiotikatherapie entziehen, könnten dies möglicherweise hoffnungsvolle Ansätze sein. In diese Richtung geht auch die Beobachtung, dass uropathogene Keime offenbar Probleme haben, in das Urothel einzudringen, wenn der cAMP-Spiegel der Zellen hoch ist. So könnten möglicherweise chronische Infektionen des Harntrakts unterbunden werden, wenn die Enzymsynthese für die cAMP-Produktion angekurbelt würde. Künftige Studien müssen klären, ob diese Ansätze das Potenzial für neue Wirkstoffgenerationen haben.

(Christine Starostzik)

Internist 2011, 52:1026

S3-Leitlinie AWMF-Register-Nr. 043/044

Weitere Informationen finden Sie im Dossier „Harnwegsinfektion" unter http://www. springermedizin.de/uro-harnwegsinfektion 